\title{
Study of Functional and Radiological Outcome in Subtrochanteric femoral Fractures Fixed with Reconstruction Nail
}

\author{
Authors
}

\section{Surendher Kumar R., Ashish Jose, Krishnagopal R., Sandeep MMR}

Department of Orthopaedics, Mahatma Gandhi Medical College \& Research Institute, Pondicherry, India

ABSTRACT
Sub-trochanteric femoral fractures present a challenging situation to the orthopaedic surgeons. There are
many implants for fixation of subtrochanteric fractures. In this study we analyze the functional and
radiological outcome of subtrochanteric fractures treated by Reconstruction nails. Irrespective of the
implant used, it is important to get a good medial bone support by anatomical reduction, with cortical bone
continuity, to prevent varus malunion. We concluded that Reconstruction nail is an ideal implant for
Subtrochanteric fractures.
Key Words: Subtrochanteric fracture, Reconstruction nail.

\section{INTRODUCTION}

Sub-trochanteric femoral fractures present a challenging situation to the Orthopaedic surgeons. It has long been recognized as one of the most difficult fractures to treat and the reported mortality ranges from $8.3 \%$ to $20.9 \%{ }^{1,2}$ These fractures have a bimodal age distribution and very different mechanisms of injury. ${ }^{3,4,5,6}$ Younger age groups typically sustain these fractures as a result of high energy trauma and are often associated with other fractures, whereas in older age groups, these fractures are seen with low velocity trauma. These fractures are reported in children also. $3,6,7$

Subtrochanteric fractures are notorious for high complication rates and difficulty in treatment. ${ }^{8}$ The reasons being:

- Majority of fractures are unstable

- Powerful muscular attachment to both fragments, pulling them apart, making a stable fixation difficult.

- It is a junctional zone between cortical and cancellous bone and having less blood supply leading to delayed healing.

Complications like malunion, nonunion and implant failure are high due to the above said reasons and also due to the biomechanically asymmetrical loading pattern in this region. ${ }^{1,2}$ Restoration of femoral length and rotation and correction of femoral head and neck angulation to restore adequate abductor tension and strength are essential to restoring maximal ambulatory capacity. There are many implants for fixation of subtrochanteric fractures. In this study we analyze the functional and radiological outcome of subtrochanteric fractures treated by Reconstruction nails. 


\section{AIM}

To analyse the functional and radiological outcome of Subtrochanteric femoral fractures treated with Reconstruction nail.

\section{MATERIALS AND METHODS}

This is a retrospective and prospective study. We had 21 patients with subtrochanteric fractures treated by reconstruction nail from January 2012 to June 2014. We analysed these 21 patients regarding functional and radiological out come and complications.

\section{Inclusion criteria:}

All traumatic subtrochanteric fracture treated with Reconstruction nail were included in the study. (Fig.1)

\section{Exclusion criteria:}

Subtrochanteric fractures treated with other implants, pathological fractures and open fractures were excluded from this study.

\section{Mode of Injury:}

1. Road Traffic Accident (16 patients)

2. Fall from height (3 patients)

3. Trivial fall (2 patients)

All the 21 patients were treated with reconstruction nailing.

\section{POST OPERATIVE PROTOCOL}

1. Sit propped up and do active quadriceps exercise on 1st post-operative day (POD).

2. Made to stand on the 2nd POD.

3. Partial weight bearing from 3rd POD (protected).

4. Sutures removed on $12^{\text {th }}$ POD.

5. Full weight bearing once there is radiological signs of union.

6. Assessment functionally \& radiologically at 6 weeks, 12 weeks, monthly until fracture union and every 6 months there after. (Figs. 2,3,4)

\section{SCORING SYSTEM}

All patients were followed up until fracture union occurred and we used the modified scoring system of Schatzker and Lambert which was originally used for distal femoral fracture and subsequently modified and used for proximal femoral fractures.

Schatzker and Lambert ${ }^{`} 79$

Excellent

Flexion loss less than 10 degrees

No Varus / Valgus / rotation deformity

No pain 


\section{JMSCR Vol.||03||Issue ||08||Page 6948-6956||August}

Good

Not more than any one of

Fair

Not more than any two of

Poor

Flexion less than 90 degrees

Varus / Valgus more than 15 degrees

Disabling pain irrespective of radiological union

According to Seinsheimer's Classification

- Type I - nil

- Type II A - 1 Patient

- $\quad$ Type II B - 3 Patients

- Type II C - 2 Patients

- Type III A - 4 Patients

- Type III B - 4 Patients

- Type IV - 5 Patients

- Type V - 2 Patients

\section{RESULTS}

Duration of follow up: 6 months to 3 years

Mean follow up: 9 months

Based on the scoring system, the results of our study are

Excellent : 6 patients

Good : 9 patients

Fair : 4 patients

Poor : 2 patients

Union rate:

Recon nail - $94.8 \%$

Union time:

Closed Reduction- 16 weeks (13 patients)

Open Reduction- 17.5 weeks (8 patients)

Varus malunion:

4 patients $(19.04 \%)$

Shortening:

4 patienets $(19.04 \%)$

Infection:

1patient $(4.7 \%)$
Shortening more than $1.2 \mathrm{cms}$

Varus / Valgus less than 10 degrees

Flexion loss more than 20 degrees

Minimal pain 
Non union:

- nil

Delayed union:

1 patient (4.7\%)

Implant Failure:

Nil

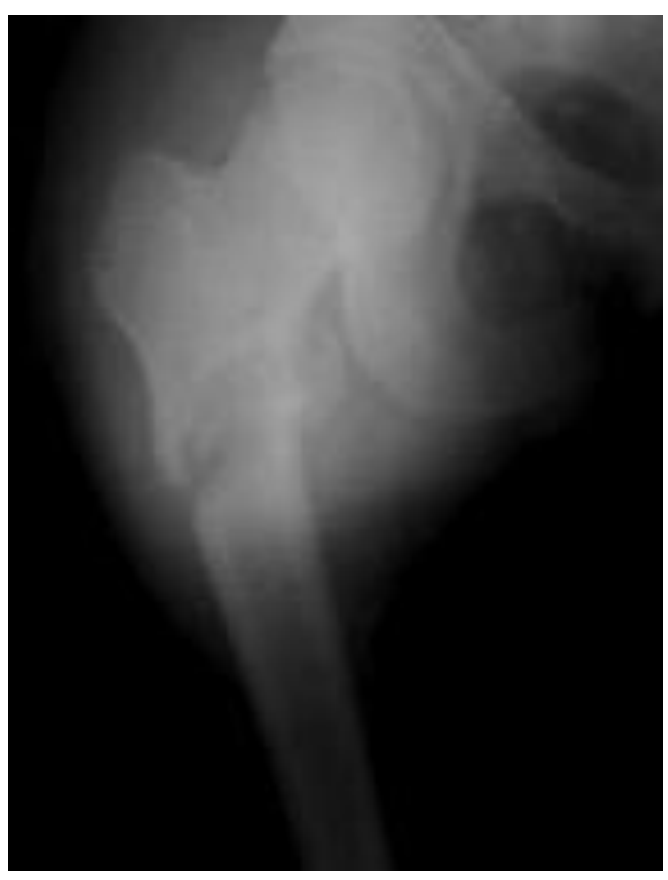

Fig. 1 : Pre-operative X-Ray

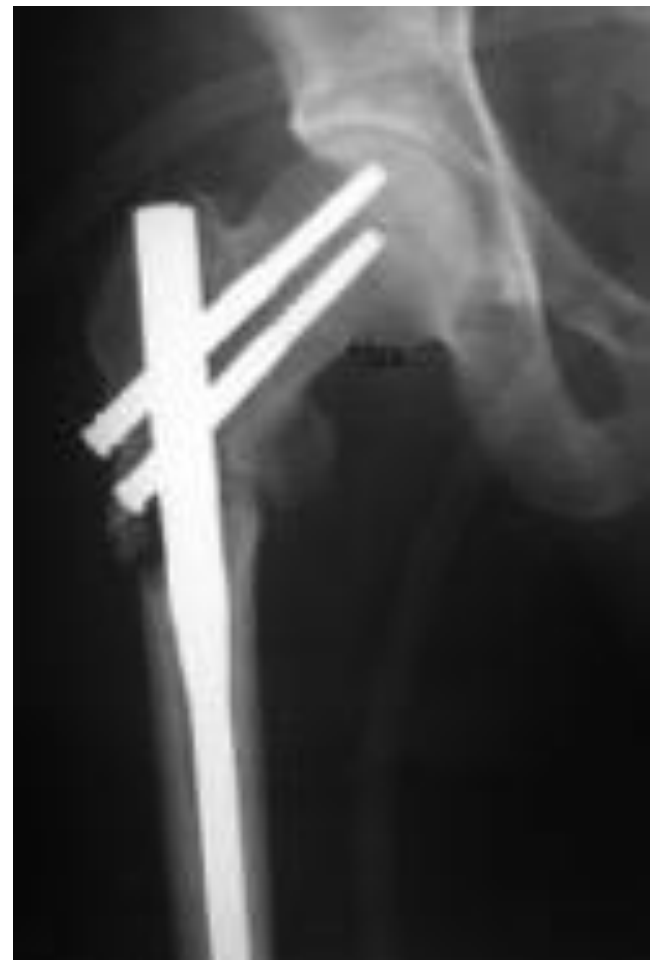

Fig. 2 : 6 weeks follow up X-Ray 


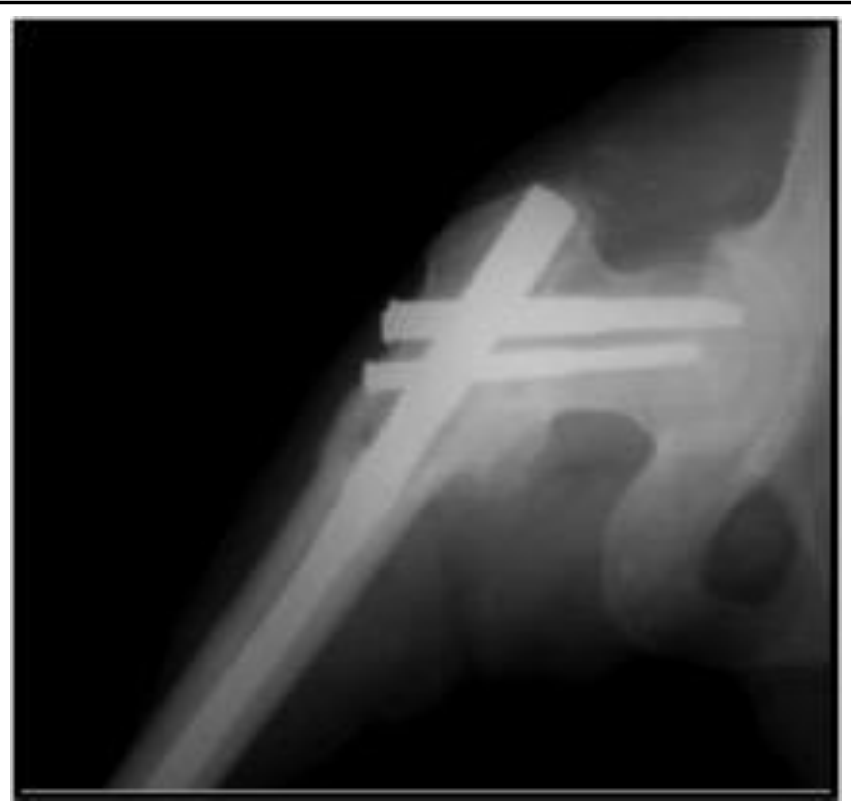

Fig.3 : 12 weeks follow up X-Ray

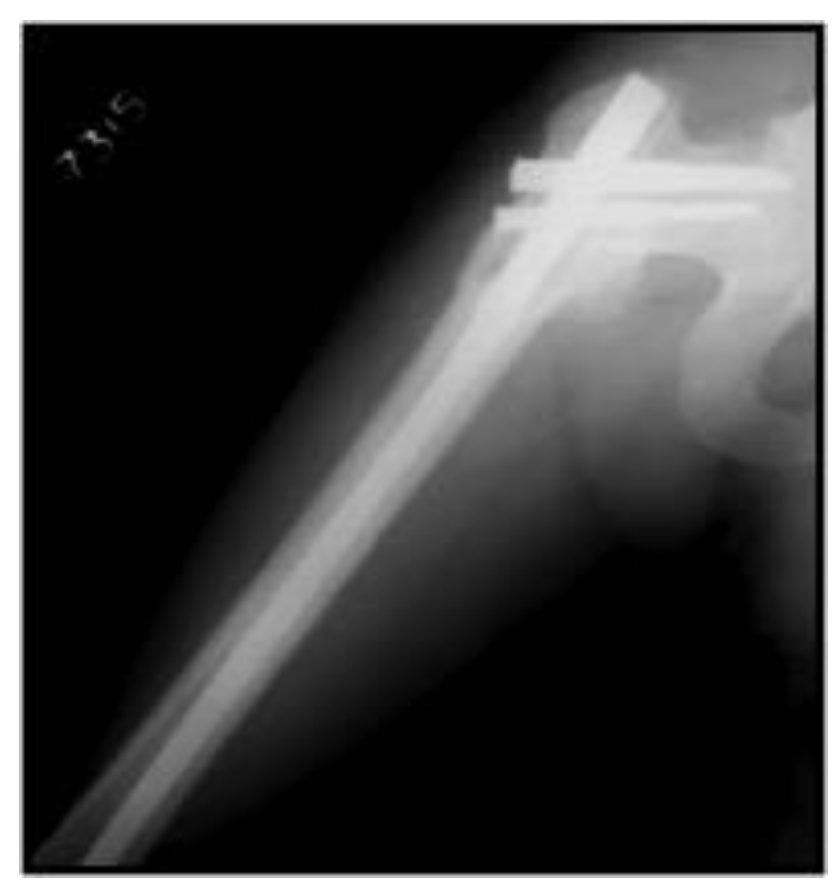

Fig. 4 : 6 months follow up X-Ray 


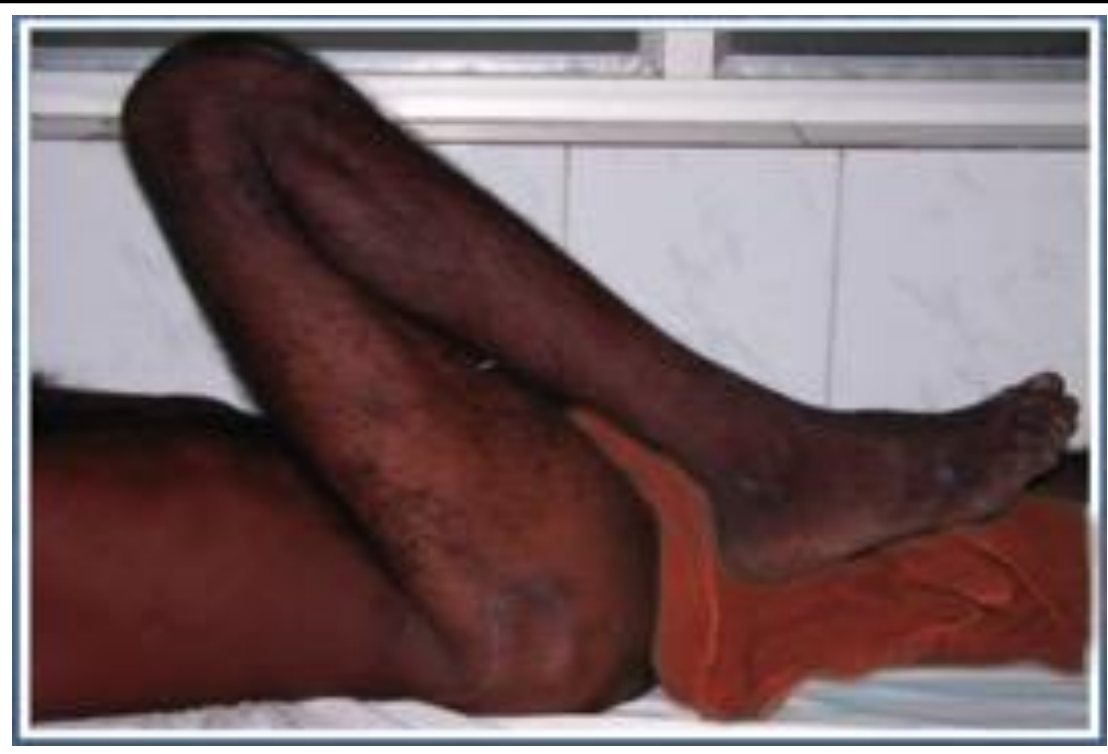

Fig.5 : Full range of hip flexion

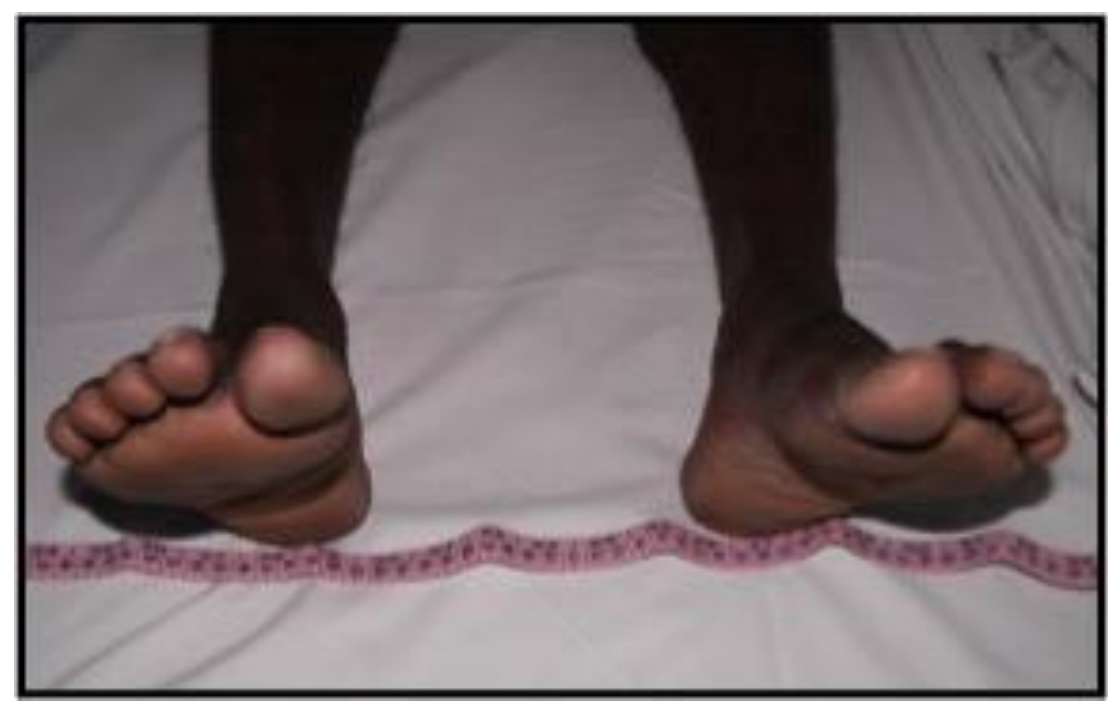

Fig.6 : No shortening

\section{DISCUSSION}

Subtrochanteric fractures account for about 5-20\% of all hip fractures. They are one of the most difficult fractures to treat. It is considered difficult because of the powerful muscles attached in this region , ${ }^{3,6}$ and the biomechanics of this segment of femur which is subjected not only to axial loads of weight bearing but also to tremendous bending forces because of eccentric load application on the femoral head. ${ }^{4}$

Once the fracture is fixed these muscular forces act on the implant causing undue stress to the implant. ${ }^{9,10}$ Moreover subtrochanteric area has an assymetrical loading pattern with medial cortex in compression (1200 $\left.\mathrm{lbs} / \mathrm{inch}^{2}\right)$ and lateral cortex in tension $\left(1000 \mathrm{lbs} / \mathrm{ich}^{2}\right) .{ }^{11}$ Frankel and Burstein, studying the effects of stress on proximal femoral fixation devices in patients during bed rest, demonstrated that significant forces are placed on the hip and proximal femur during hip flexion and extension and even while the patient is recumbent. $^{12}$

Further more, the cortical bone in the subtrochanteric region is less vascular than the cancellous bone in the intertrochanteric region. Therefore, the risk of healing complication is higher. ${ }^{2,3}$ 
A thorough understanding of the anatomy and biomechanics of assymetrical loading pattern is necessary to choose the modality of treatment and the implant to overcome the complication and to avoid implant failure.

\section{Fracture union Rate:}

In our study average union rate in was $94.8 \%$. One patient went in for a delayed union, which is a Seinsheimer's Type V fracture. This patient had blow out of Greater Trochanter while making an entry point to nail. In spite of following a strict postoperative protocol no signs of radiological union was noted at the end of 7 months. Secondary bone grafting was done at the 8 month and union was achieved on the $11^{\text {th }}$ month.

In our study, we achieved $94.8 \%$ union rate. French et al, ${ }^{13}$ Taylor et al, ${ }^{14}$ had reported $100 \%$ union rate in their study and Hoover et al reported $90 \%$ union rate. $^{15}$

\section{Union Time:}

In our study, we had an average union time of 16 weeks. French et al reported 13.5 weeks as his average union time in his study. ${ }^{13}$ Taylor et al reported average union time of 13 weeks in his study. ${ }^{14}$

\section{Varus Malunion:}

The angle formed by the axis of the femoral neck and femoral shaft ranges from $126^{\circ}$ to $130^{\circ}$. If the angle is decreased it is said to have varus malunion. ${ }^{6}$ The primary reason for this was failure to counteract the muscle forces acting on the proximal fragment combined with adducted position of the distal femur during portal creation. ${ }^{13}$ It is also important to get a good medial bone support by anatomical reduction with cortical bone continuity to avoid varus malunion. 5

In our study, 4 patients had varus malunion. In one of them it is attributed to not able to position the proximal fragment while creating portal for entry. In the other three patients it is attributed to inadequate medial bone support due to comminution. In our study varus malunion showed $19.04 \%$. French et al reported $21 \%,{ }^{13}$ Hoover et al reported $27 \%$ in their series. ${ }^{15}$

In Reconstruction nailing, varus malunion can be prevented if the proximal fragment is evaluated carefully, reduced and holding it with a Schanz's screw ( joy stick) while creating a portal for entry. In addition, excessive adduction of the distal fragment during reaming and nail placement should be avoided. ${ }^{13}$

\section{Iatrogenic fracture:}

In our study, iatrogenic fracture of greater trochanter occurred in 1 patient. The fracture remained reduced and had uneventful union. This is mainly a technical error and can be avoided. ${ }^{13}$ Iatrogenic fracture accounted to $4.7 \%$ in our study. French et al reported $5 \%$ of iatrogenic fracture in his series. ${ }^{13}$

\section{Implant failure:}

Our study did not account for any implant failure which is same as the literature available.

\section{Shortening:}

In our study, 4 patients had shortening. The average shortening was $1.6 \mathrm{~cm}$. All 4 patients were given heel and sole raise. In our study shortening accounted to $23.8 \%$. French et al reported $5 \%,{ }^{13}$ and Hoover et al reported $23 \%$ in their series. ${ }^{15}$

\section{Infection:}

In our study, 1 patient reported late infection after 10 months. This patient underwent reconstruction nailing by open reduction. Radiograph showed good fracture union with proximal screw loosening and back out. 
This patient was managed by implant removal and antibiotics. Infection rate in our Recon nail group was $4.3 \%$. Infection rate was nil in the study of French et al,$^{13}$ Taylor et $\mathrm{al}^{28}$, Hoover et $\mathrm{al}^{19}$.

\section{Blood loss:}

Average blood loss in our study was $400 \mathrm{ml}$. French et $\mathrm{al}^{16}$ had $340 \mathrm{ml}$, Taylor et al had $620 \mathrm{ml},{ }^{14}$ Hoover et al had $480 \mathrm{ml}$ in their study. ${ }^{15}$

\section{Scoring result:}

In our study, excellent and good result accounted to $71.42 \%$ (15 patients). (Figs. 5,6)

Fair result accounted to $19.04 \%$ (4 patients). Out of the 4 patients, the first one had flexion loss of hip more than $20^{\circ}$ and pain on walking long distances (Type V), the second patient had shortening and pain on walking long distances (Type V), the third patient had varus malunion With shortening (Type IV), the fourth patient had varus malunion with flexion loss of hip more than $20^{\circ}$.

Poor result accounted to $09.52 \%$ ( 2 patients). One of them had flexion of hip less than $90^{\circ}$, varus malunion and pain on walking (Type III A). The other patient had flexion of hip less than $90^{\circ}$, disabling pain and reported late infection at 10 months postop (Type III B).

\section{Fracture pattern:}

Type III A

In our study, we had 4 patients of Seinsheimer's Type III A fracture. Out of the 4 patients in 2 patients had excellent to good result, 1 patient had fair and 1 patient had poor result.

Type III B

In our study, we had 4 patients of Seinsheimer's Type III B fracture. Out of the 4 patients, 3 patients had excellent to good result and 1 patient had a poor result.

Type IV

In our study, we had 5 patients of Seinsheimer's Type IV fracture. Out of the 5 patients , 4 patients had excellent to good result and 1 patient had fair result.

Type V:

In our study, we had 2 patients of Seinsheimer's Type V fracture. Both the patients had fair result.

\section{CONCLUSION}

Reconstruction Nail is an ideal implant for Subtrochanteric fracture without Inter-trochanteric extension. Irrespective of the implant used, it is important to get a good medial bone support by anatomical reduction, with cortical bone continuity, to prevent varus malunion. Implant Failure rate is low in Reconstruction nail since Nail is a load sharing device. Infection rate is relatively less and Reconstruction Nailing has got very good union rate (95\%). Closed Reconstruction Nailing has got the lesser healing period (Avg-16weeks). Careful pre-operative planning, good intra-operative technique and strict post-operative protocol when followed gives good results in Subtrochanteric Femoral fractures.

\section{REFERENCES}

1. Pugh KJ, Morgan RA: A mechanical comparison of subtrochanteric femur fracture fixation. J Orthop Trauma 12: No 5: $324-329 ; 1998$.

2. Seinsheimer F: Subtrochanteric fractures of the femur. J Bone Joint Surg 60 - A: 300 - 306; 1978.

3. Asheesh Bedi, MD, T.Toan Le MD: Subtrochanteric femur fractures. Orthop Clin North Am 35: 473 $-483 ; 2004$. 
4. Kinast C, Bolhofner BR, Mast JW, Ganz R: Subtrochanteric fractures of the femur, results of 95* Condylar blade plate. Clin Orthop 238: 122 - 130; 1989.

5. Nungu KS, Olerud C, Rehnberg L: Treatment of subtrochanteric fracture with A O dynamic condylar screw. Injury; 24(2); 90 - 92: Feb 1993.

6. Sims SH: Subtrochnteric femoral fractures. Orthop Clin North Am 33: (1): 113 - 126,Jan 2002.

7. Tidermark J, Hamberg P: Extramedulary fixation of 107 subtrochanteric fractures. Acta Orthop Scand 70; (5); 459-466; 1999.

8. Schatzker J: Text book of "Rationale of operative orthopaedics". 1: $349-386$.

9. Cochran GVB: Implantation of strain gauges in bone in vivo. J Biomechanics 5: 119; 1972.

10. Feilding JW, Cochran GVB, Zickel RE: Biomechanical characteristics and surgical management of Subtrochanteric fractures. Orthop Clin North Am 5: 629-650;1974.

11. Koch JC: The laws of bone architecture. Am J Anat 21: 177 - 298; 1917.

12. Frankel V, Burstein A: Orthopaedic biomechanics. Lea and Febiger Philadelphia Lea\& Febiger; 1970.

13. French BG: Use of an interlocked cephalomedullary nail for subtrochanteric fracture stabilization. Clin Orthop 348: 95 - 100; 1998.

14. MAJ Taylor DC: treatment of comminuted subtrochanteric femoral fractures in a young population with a reconstruction nail. Mil Med 161: 735 - 738; Dec 1996.

15. Hoover GK, Browner BD: Initial experience with a second generation locking femoral nail. The Russell - Taylor reconstruction nail. Contemp Orthop 23: 199 - 208; 1991. 